\begin{tabular}{|c|l|}
\hline Title & $\begin{array}{l}\text { Effects of surface states and Si-interlayer based surface passivation on GaA s quantum wires grown by selective } \\
\text { molecular beam epitaxy }\end{array}$ \\
\hline Author(s) & Shiozaki, Nanako; Sato, Taketomo; Hasegawa, Hideki \\
\hline Citation & $\begin{array}{l}\text { Journal of V acuum Science \& Technology B: Microelectronics and Nanometer Structures, 23(4), 1714 1721 } \\
\text { https:/doi.org/10.1116/1943446 }\end{array}$ \\
\hline Issue Date & 2005-07 \\
\hline Doc URL & http://hdl.handle.net/2115/8382 \\
\hline Rights & ○ 2005 A merican Vacuum Society \\
\hline Type & article \\
\hline File Information & TSA TO8.PDF \\
\hline
\end{tabular}

Instructions for use 


\title{
Effects of surface states and Si-interlayer based surface passivation on GaAs quantum wires grown by selective molecular beam epitaxy
}

\author{
Nanako Shiozaki, ${ }^{\text {a) }}$ Taketomo Sato, and Hideki Hasegawa \\ Research Center for Integrated Quantum Electronics (RCIQE) and Graduate School of Information Science \\ and Technology, Hokkaido University, North 13, West 8, Kita-Ku, Sapporo 060-8628, Japan
}

(Received 23 January 2005; accepted 2 April 2005; published 25 July 2005)

\begin{abstract}
Effects of surface states and surface passivation on photoluminescence (PL) properties of GaAs quantum wires (QWRs) are investigated. QWR samples were grown on (001) and (111)B substrates by the selective molecular beam epitaxy (MBE) method. For surface passivation, an ultrathin (about $1 \mathrm{~nm}$ ) Si interface control layer (Si ICL) was grown by MBE as an interlayer. In both of the selectively grown QWRs on (001) and (111)B substrates, the PL intensity reduced exponentially with reduction of their wire-to-surface distance, being coexistent with a more gradual reduction due to carrier supply reduction. The exponential reduction was explained in terms of interaction between surface states and quantum confined states leading to tunneling assisted nonradiative recombination through surface states. Surface passivation by the Si-ICL method almost completely recovered PL intensities not only for QWRs on the (001) substrate, but also for QWRs on the (111)B substrate. (C) 2005 American Vacuum Society. [DOI: 10.1116/1.1943446]
\end{abstract}

\section{INTRODUCTION}

In recent years, the semiconductor nanotechnology has made tremendous progress, and varieties of approaches are now available for formation of artificial quantum nanostructures such as quantum wires (QWRs) and quantum dots (QDs). In particular, selective molecular beam epitaxy (MBE) or metalorganic vapor phase epitaxy growth of III-V material-based QWRs and QDs on patterned or masked substrates $^{1-5}$ is one of the most promising approaches due to the following features. (1) Size and position of the nanostructure can be controlled. (2) Sizes smaller than lithography sizes can be obtained. (3) Lithography size fluctuations do not propagate to nanostructure interfaces. (4) Nanostructures are completely embedded in crystals by high quality interfaces with strong quantum confinements.

Thus, it is likely that QWRs and QDs by such approaches may become basic building blocks for next generation electronics and photonics. In fact, our group has proposed a hexagonal binary decision diagram (BDD) quantum circuit approach $^{6-8}$ for large scale integration of quantum devices. Here, selectively MBE grown quantum wire networks are controlled by nanometer-scale Schottky gates to form single electron transistor or quantum wire transistor switches and to perform logic functions based on the BDD architecture.

However, one of the remaining key processing issues is the surface state problem, since III-V materials possess highdensity surface states, and their effects increase in nanostructures due to the increased surface-to-volume ratio. In the case of the selective growth, nanostructures are embedded in high quality barrier crystals. However, they usually lie near surfaces in advanced device designs. For example, the gate should lie near to the quantum structures for an efficient gate control in transport devices. Thus, it is likely that surface

a) Author to whom correspondence should be addressed; electronic mail: shiozaki@rciqe.hokudai.ac.jp states affect properties of quantum confined states. Expected unfavorable effects are (1) tunneling assisted carrier leakage and resultant nonradiative recombination due to interaction between surface states and quantum confined states, (2) carrier depletion in nanostructures due to strong surface Fermi level pinning, (3) reduction of gate control efficiency due to Fermi level pinning on the free surface near the gate periphery, ${ }^{9}$ and (4) shift of threshold voltage and related long-term instability due to charge-discharge transients of surface states.

In spite of such importance, however, only limited works have been done so far to clarify the effect of surface states on quantum confined states. Namely, behavior of AlGaAs/GaAs quantum wells (QWs), ${ }^{10,11}$ etched AlGaAs/GaAs QWRs, ${ }^{12}$ and InAlAs/InGaAs/InP QWRs (Ref. 13) has been investigated, using the photoluminescence (PL) technique as a probe. In all cases, drastic reduction of PL intensity was observed in near-surface nanostructures. However, its mechanism as well as a method to avoid this effect has not been amply established so far.

The purpose of this article is to investigate the effects of surface states and surface passivation on PL properties of completely embedded AlGaAs/GaAs QWRs grown on (001) and (111)B substrates by the selective MBE method developed by the authors' group. ${ }^{4,5}$ The reason for use of the unconventional (111)B substrate is the following. Due to its sixfold symmetry, the wires grown on the (111)B substrate are inherently more suitable for the hexagonal BDD logic circuits where the basic node device has a threefold symmetry. For surface passivation, a Si-interlayer based technique also developed by the authors' group ${ }^{14,15}$ was used. This method includes MBE growth of an ultrathin (about $1 \mathrm{~nm}$ ) Si interface control layer (Si ICL) to terminate surface dangling bonds.

In both types of QWRs selectively grown on (001) and (111)B substrates, the PL intensity reduced exponentially 


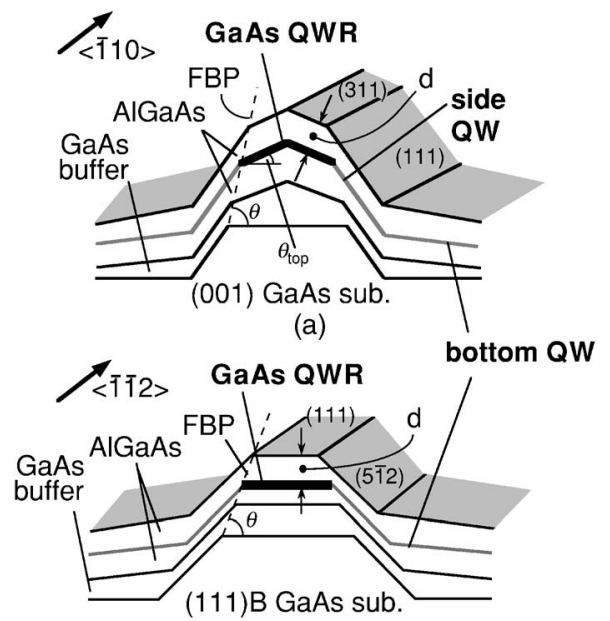

(b)

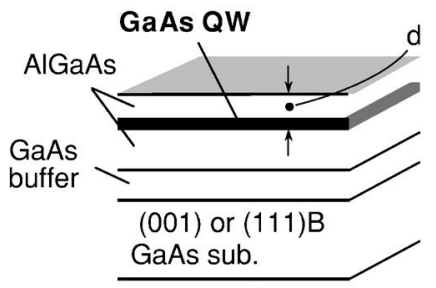

(c)

FIG. 1. Cross-sectional structures of QWR samples grown on (a) (001) and (b) (111)B GaAs substrates. (c) Reference QW sample grown on planar substrate.

with reduction of their wire-to-surface distance, being coexistent with a more gradual reduction due to carrier supply reduction. The exponential reductiont was explained in terms of interaction between surface states and quantum confined states leading to nonradiative recombination through surface states. Surface passivation by the Si-ICL method almost completely recovered PL intensities.

\section{EXPERIMENT}

\section{A. Selective MBE growth of QWR samples}

The cross-sectional structures of QWR samples selectively grown on (001) and (111)B substrates are shown in Figs. 1(a) and 1(b), respectively. For their growth, the sequence developed by the authors' group ${ }^{4,5}$ was used. Namely, an array of mesa stripes running along $<-110\rangle$ direction and $\langle-1-12\rangle$ direction was formed on (001) and (111)B GaAs substrates by the electron beam lithography and wet chemical etching. Then, GaAs buffer ridge structures were grown by MBE. Subsequently, by growing $\mathrm{Al}_{0.3} \mathrm{Ga}_{0.7} \mathrm{As} / \mathrm{GaAs} / \mathrm{Al}_{0.3} \mathrm{Ga}_{0.7} \mathrm{As}$ structure on ridges by MBE, completely embedded QWRs were formed due to selective growth mechanism. For the purpose of monitoring, a planar substrate was placed next to the patterned substrate, and this led to growth of QW samples schematically shown in Fig. 1(c). In this study, the $\mathrm{Al}$ composition in the AlGaAs layers was fixed to 0.3 , and thus $\mathrm{AlGaAs}$ in this article always means $\mathrm{Al}_{0.3} \mathrm{Ga}_{0.7} \mathrm{As}$.

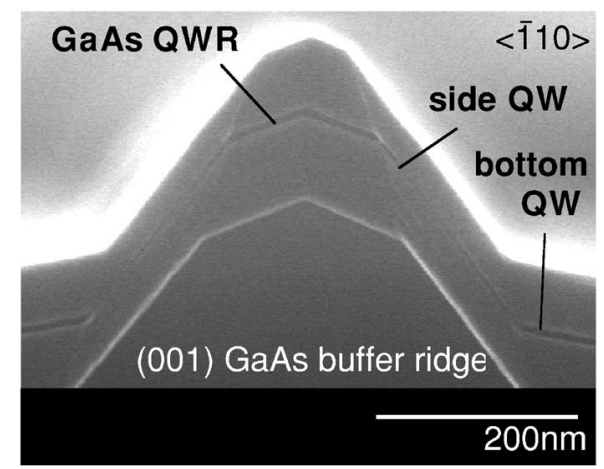

(a)

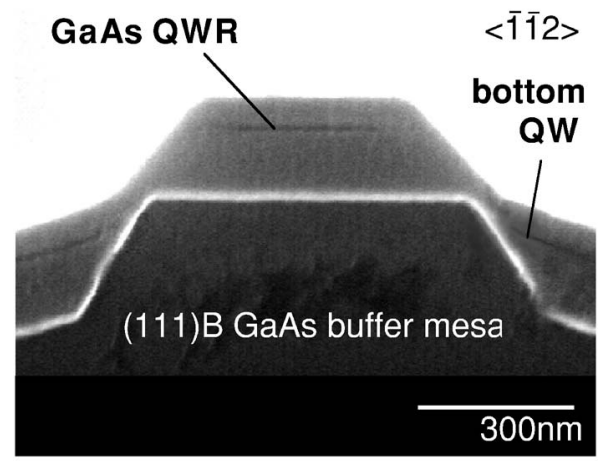

(b)

FIG. 2. Cross-sectional SEM images of selectively grown GaAs QWRs on (a) (001) and (b) (111)B substrates.

Based on the recent study, ${ }^{16}$ the lateral wire width, $W$, and the vertical wire height, $H$, are kinetically controlled by the growth conditions, using the following equations:

$$
\frac{W}{W_{0}}=1-\beta \cdot t, \quad \beta=2 \frac{g_{\text {top }}}{\mathrm{W}_{0}\left(\tan \theta-\tan \theta_{\mathrm{top}}\right)}
$$

and

$$
H=g_{\text {top }} t,
$$

where $W_{0}$ is the initial mesa width on the buffer ridge, $g_{\text {top }}$ is the vertical growth rate of the top facet, $t$ is the growth time, and $\theta$ and $\theta_{\text {top }}$ are the angle of the facet boundary plane (FBP) ${ }^{16}$ and the top facet angle with reference to Figs. 1(a) and 1(b), respectively. FBPs play important roles in determining the height and width of the QWR. ${ }^{16}$ The typical values of $H$ and $g_{\text {top }}$ used in the present study were $90 \mathrm{~nm}$ and $770 \mathrm{~nm} / \mathrm{h}$ for (001) QWR samples and were $130 \mathrm{~nm}$ and $740 \mathrm{~nm} / \mathrm{h}$ for (111)B QWR samples, respectively.

Examples of cross-sectional images of the grown samples measured by a JEOL scanning electron microscope (SEM) are shown in Figs. 2(a) and 2(b) for a (001) QWR sample and a (111)B QWR sample, respectively. Existence of completely embedded GaAs QWRs can be clearly identified.

\section{B. Method of characterization}

In order to investigate possible effects of surface states on the quantum confined states, PL properties of the QWR 


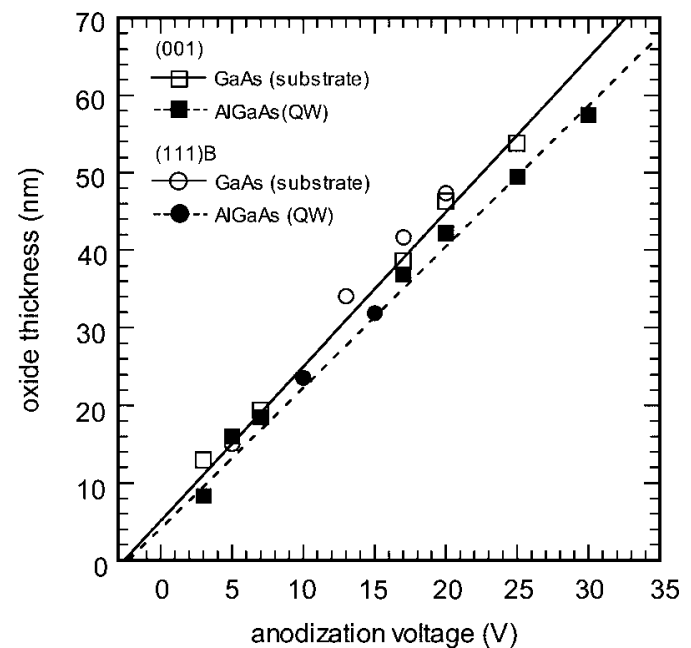

FIG. 3. Thickness of anodic oxide as a function of anodization voltage.

samples having different values of the QWR-to-surface distance, $d$, or the thickness of the $\mathrm{Al}_{0.3} \mathrm{Ga}_{0.7} \mathrm{As}$ top barrier layer, as defined in Figs. 1(a) and 1(b), were compared on the basis of previous similar works on QWs. ${ }^{10,11}$ The value of $d$ was changed systematically, either by changing the growth thickness of the top AlGaAs layer in the MBE run, or by etching the top AlGaAs layer of the QWR sample by wet chemical etching or anodic etching.

Particularly, the anodic etching was found to be useful for performing precisely controlled etching in the nanometer range. This is because the thickness of the anodic oxide is basically determined by the anodization voltage. For this purpose, an anodic oxide was formed in a mixed solution of tartaric acid and propylene glycol (AGW electrolyte) ${ }^{17}$ and subsequently it was dissolved in a hydrochloric acid solution.

Basic anodic oxidation experiments in the nanometer range were carried out on AlGaAs surfaces of the (001) and (111)B AlGaAs/GaAs/AlGaAs QW samples grown by MBE on GaAs substrates as well as on (001) and (111)B GaAs substrate surfaces themselves. In all cases, the surface layer was $n$ type. Flat surfaces were used, because the oxide thickness on planar surface can be accurately measured by the ellipsometry technique and by the atomic force microscopy (Digital Instruments Nanoscope IIIa) measurements, as compared with complex structures having ridges. For ellipsometric measurement, we used the published semiconductor optical data. ${ }^{18}$

The measured values of oxide thickness are summarized in Fig. 3. It is seen in Fig. 3 that linear relationships are maintained down to very small values of thickness in all cases. The reason why four lines cross at the negative values of the anodization voltage is due to the photovoltaic effect caused by light illumination of the surface during anodization of $n$-type materials to supply holes required for the anodic oxidation reaction. The measured average oxidation rates were slightly different for GaAs and AlGaAs, being $2.0 \mathrm{~nm} / \mathrm{V}$ for (001) GaAs, $2.0 \mathrm{~nm} / \mathrm{V}$ for (111)B GaAs, $1.8 \mathrm{~nm} / \mathrm{V}$ for (001) AlGaAs, and $1.8 \mathrm{~nm} / \mathrm{V}$ for (111)B AlGaAs surfaces, respectively. The value of $2.0 \mathrm{~nm} / \mathrm{V}$ for
(001) GaAs surface was in good agreement with the previous study, ${ }^{17}$ although the previous study was carried out in a much larger oxide thickness range. After establishing the basic data, the anodic etching technique was applied to etching of the ridge QWR samples. From the cross-sectional SEM observation, it was found that the etching rates of the top AlGaAs barrier layers of QWRs were the same with those of the planar samples.

QWR samples were characterized by PL measurements performed at $25 \mathrm{~K}$ under an Ar laser excitation, using a Si charge coupled device (CCD) photodetector (Princeton Instruments Inc., model LN/CCD-1024-EHR/1). For assignments of PL peaks, spatially resolved cathode luminescence (CL) measurements were also carried out, using a JEOL JSM-5410 SEM with the Oxford MonoCL 302 m monochromator.

\section{Method of Si-ICL based passivation}

In an attempt to reduce surface state effects, the Si ICL based surface passivation process developed by the authors' group was applied to QWR samples, using the ultrahigh vacuum (UHV) multichamber system. The details of the process for application to the technologically important (001) surfaces have already been described in Refs. 14 and 15. However, since such a passivation technique has not been applied so far to the (111)B surface, a basic study was made in this study by the in situ X-ray photoelectron spectroscopy (XPS) technique, using Perkin-Elmer PHI 1600 with a monochromatic Al $K \alpha$ source at $1486 \mathrm{eV}$. The electron energy detected by the spectrometer was corrected by using the gold standard samples before the experiments.

The process sequence that was taken to apply the Si ICL method to (001) and (111)B QWR samples was the following. After conventional oxide removal in the MBE chamber from the surface of the air-exposed QWR sample, an ultrathin silicon called Si ICL was grown by MBE on the surface of the top AlGaAs barrier layer of the sample in order to terminate the dangling bonds of the surface. More specifically, about 1-nm-thick Si layer was grown on the QWR surface at a substrate temperature of $300{ }^{\circ} \mathrm{C}$, using a Si beam from a Si $K$ cell held at $1215{ }^{\circ} \mathrm{C}$. Subsequently, the top of the Si ICL was partially converted into a SiN layer by direct nitridation at room temperature, using a nitrogen radical beam, in order to terminate the dangling bonds on the surface of the Si ICL. After this, the sample was taken from the UHV system and exposed to air in order to investigate the effects of the surface passivation on PL properties of the QWR sample.

Additionally, it has been recently found ${ }^{15}$ on the (001) surface that the process is more effective on group-III stabilized surfaces rather than on group-V stabilized surfaces. In the present case of QWR samples, attempts to realize groupIII stabilized surfaces were also made by irradiating the Al$\mathrm{GaAs}$ surface with a small amount of $\mathrm{Ga}$ or $\mathrm{Al}$ flux before $\mathrm{Si}$ growth. 


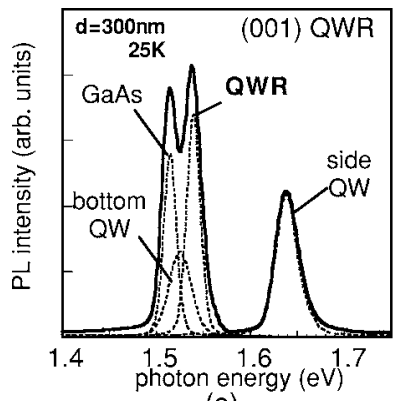

(a)

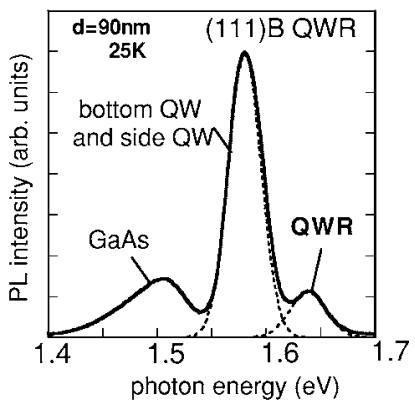

(b)

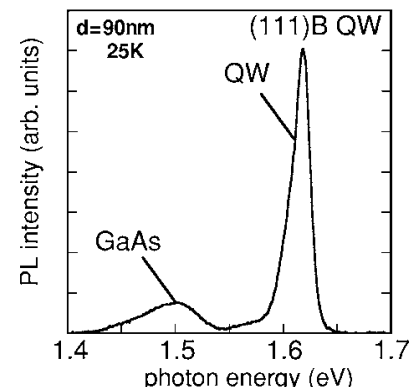

(c)

FIG. 4. (a) PL spectra from a (001) QWR sample, (b) those from a (111)B QWR sample, and (c) those from a reference QW sample on (111) B substrate.

\section{RESULTS AND DISCUSSION}

\section{A. Results of PL measurements}

Typical PL spectra obtained on QWR samples grown on (001) and (111)B are shown in Figs. 4(a) and 4(b), respectively. For comparison, the PL spectra taken on the reference QW sample formed on (111)B substrate is shown in Fig. 4(c). As shown by dashed curves obtained by Gaussian deconvolution of peaks, PL peaks due to the QWR and those coming from other parts could be clearly identified on the PL spectra of QWR samples by correlating the PL peak energies with the spatially resolved CL scans taken on the same sample. To show this, a plan view SEM image and CL data taken on the (001) QWR sample are shown in Figs. 5(a)-5(c) and those taken on the (111)B QWR sample are shown in Figs. 5(d)-5(f), respectively. In the case of the (001) QWR sample, additional PL peaks coming from bottom and side QWs were also observable in agreement with our previous study. ${ }^{4}$ On the other hand, peak energies of bottom and side QW peaks seem to overlap in the case of the (111)B QWR sample, as indicated by the CL scan shown in Fig. 5(f).

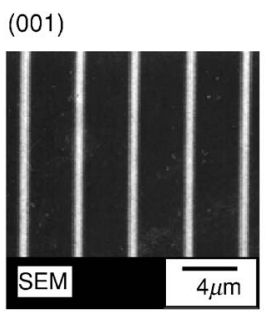

(a)

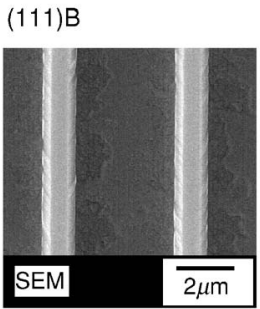

(d)

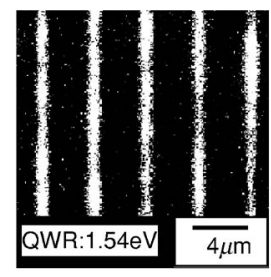

(b)

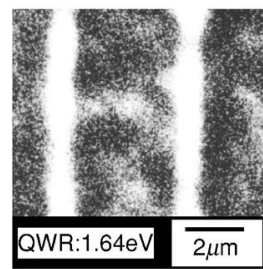

(e)

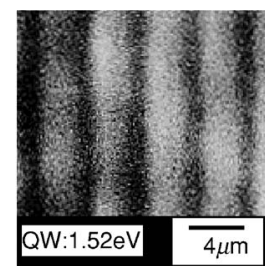

(c)

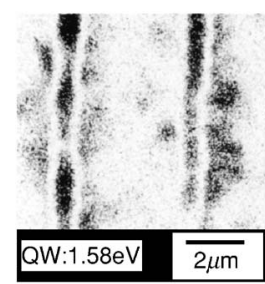

(f)
FIG. 5. (a)-(c) SEM images and CL data from the (001) QWR sample and (d)-(f) SEM images and CL data from the (111) B QWR sample.

After establishing origins of PL peaks, we first noted in Figs. 4(a) and 4(b) that the positions of the PL peaks were very different between the (001) and (111)B QWR samples in spite of the similarity of the values of $H$ and $g_{\text {top. }}$. A closer SEM investigation showed that this difference mainly came from the fact that the vertical thickness of the QWR was considerably different, reflecting the difference in the amount of wire material supplied as well as the difference in the growth selectivity of the top facet. Namely, the vertical thickness of the (001) QWR sample was $10 \mathrm{~nm}$, whereas that of the (111)B QWR sample was $4 \mathrm{~nm}$. Since lateral confinement is still rather weak in both samples with large values of $W$, this difference of the vertical thickness mainly caused the difference of the energy positions of both PL and CL emission peaks. The emission peak energy of $1.54 \mathrm{eV}$ for the (001)QWR sample and that of $1.64 \mathrm{eV}$ for the (111)B QWR sample were in good agreement with the theoretical values calculated on computer, using the observed cross-sectional structures and dimensions of two QWRs.

As for the PL intensity, the PL peak from the QWR in the (001) QWR sample was sharp and high as compared with other peaks. On the other hand, the intensity of the QWR peak of the (001) QWR sample may seem rather too small as compared with intensity of the QW peak of the same sample. In fact, the integrated intensity of the QWR peak at $1.64 \mathrm{eV}$ was approximately one third of that of the QW peak at 1.58 $\mathrm{eV}$. This difference is partly due to overlap of the side QW peak and the bottom QW peak, as mentioned earlier. Another reason was that, as compared with the (001) QWR sample, the bottom and side QWs in the (111)B QWR sample, having the same PL peak energy, were found to occupy a much larger area than that of the QWRs on the same sample, as directly confirmed by the SEM images. It is also noted that QWR peaks in both sample have peak heights similar to those of the GaAs peak coming from inside, which may be a better reference for comparison. As for the peak width, the 


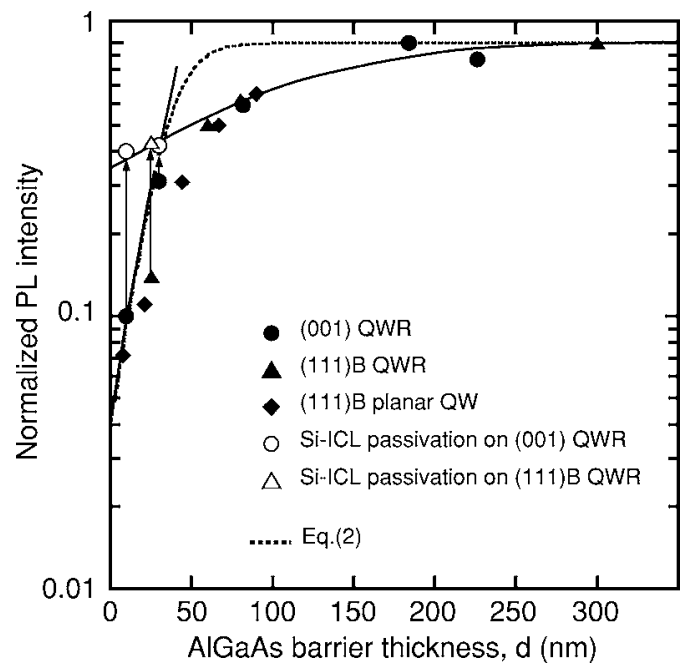

FIG. 6. Normalized PL intensities from various QWR samples and a reference QW sample as a function of the top AlGaAs barrier thickness, $d$.

full width at half maximum values of the QWR peaks are nearly the same with that of the reference QW shown in Fig. 4(c).

Finally, in order to see the effect of the QWR-to-surface distance, $d$, on the PL properties of the QWR samples, the intensities of the QWR PL peaks, which were determined by Gaussian deconvolution of peaks, are plotted in Fig. 6 as a function of the distance, $d$. The intensity is normalized by the PL intensity obtained at $d=300 \mathrm{~nm}$. For the purpose of comparison, the data taken on reference QW samples formed on (111)B substrates are also plotted. It is clearly seen that data points collected on two types of QWR samples as well as those from the QW sample showed nearly the same behavior where two tendencies are intermixed as indicated by two solid curves as eye guides. Namely, starting from large values of $d$, the PL intensity showed a gradual decrease with reduction of $d$, and this is then rather abruptly followed by a very sharp exponential decrease approximately below $d$ $=30 \mathrm{~nm}$. The observed behavior is very similar to that of InP-based QWRs. ${ }^{13}$

\section{B. Mechanism of PL intensity reduction}

Out of the observed two different $d$-dependencies of PL intensity shown in Fig. 6, the part showing sharp exponential drop of the PL intensity for values of $d$ below $30 \mathrm{~nm}$ can be explained in terms of tunneling assisted surface recombination of photo-generated carries through surface states at the QWR surface. To show this, the present embedded QWR structures [Figs. 7(a) and 7(b)], are replaced by a simple model shown in Fig. 7(c). Here, each QWR is represented by a rectangular stripe with a width, $W$, and a thickness, $t$. To proceed further, we assumed that rates of the radiative recombination and nonradiative surface recombination processes are both limited by supply of minority carriers (holes) under weak excitation, and that the QWR width, $W$, is smaller than the diffusion length of minority carriers in the QWR. Then, it is easy to show that the PL intensity, $I_{\mathrm{PL}}$, from

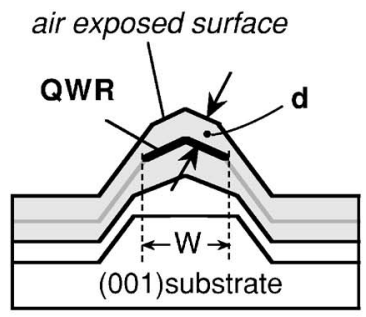

(a)

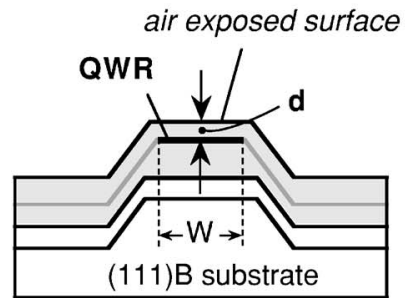

(b)

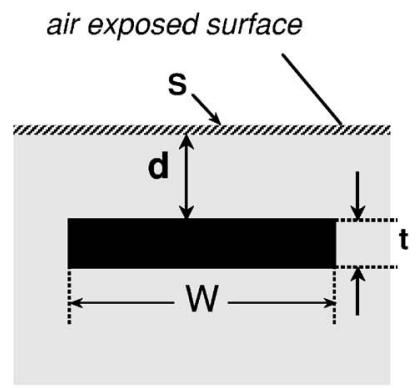

(c)

FIG. 7. Schematic cross sections of (a) (001) QWR, (b) (111)B QWR, and (c) a simple model for analysis of their PL response.

the embedded QWR is given by the following simple equation:

$$
I_{\mathrm{PL}}=A \frac{1}{1+\frac{\tau_{\mathrm{RAD}} S}{t} \exp (-\alpha d)} \phi,
$$

where $S$ is the effective surface recombination velocity measured directly on the QWR surface when it is exposed to air, $\tau_{\mathrm{RAD}}$ is the radiative lifetime of minority carriers in the QWR, $\alpha$ is the tunneling decay rate of the minority carrier wave function within the barrier layer, $\phi$ is the incident photon excitation flux intensity, and $A$ is a constant related to minority carrier supply from barrier region and photon collection efficiency for both QWR structures. In spite of simplifications of QWR structures and of various assumptions, the earlier simple equation gives a constant PL intensity at large values of $d$ as well as rapid exponential reduction of PL intensities for values of $d$ below a certain value, thus, reproducing the gross behavior of the experimental data.

From a rigorous point of view, however, such a simple treatment may look like nonsense, since a large band bending is expected to be present near the air-exposed surface of the QWR sample in the dark, similar to usual cases of airexposed III-V surfaces. In fact, XPS measurements done in this study detected a large band bending on the air exposed 
(111)B GaAs surface, as described later. Thus, an exact analysis of the recombination dynamics related to the PL from a QWR sample should be a very complicated twodimensional one which requires a self-consistent computer solution for band bending, tunneling, minority and majority carrier flows, and various bulk and surface recombination processes. Previously, such a computer analysis was made by our group on PL intensities of AlGaAs/GaAs QWs, ${ }^{19}$ using a one-dimensional program. Extension of such an analysis to the present QWR case is obviously beyond the scope of this article. However, on the basis of our previous experience on a rigorous analysis of the $\mathrm{QW}$, the earlier simple analysis seems to be acceptable as a first-order approximation, if $S$ is interpreted as an effective surface recombination velocity measured at the edge of the depletion layer caused by the band bending which is often referred to as the dead layer. ${ }^{10,20}$ According to the rigorous analysis, the magnitude of band bending is actually dependent on the excitation intensity, and quickly reduces to zero with the increase of excitation intensity. It should also be noted that the tunneling distance required for photoexcited carriers to recombine through surface states is always $d$, being independent of band bending, but the portion of the surface state distribution taking part in surface recombination is very much affected by band bending.

Bearing this in mind, the result of fitting of the above simple equation to the experimental data is shown by the dashed curve in Fig. 6. The dashed curve could very well be fitted to the exponentially decaying part of the data. Using a value of $\tau_{\mathrm{RAD}}=1 \mathrm{~ns}$, parameter values of $S=1 \times 10^{4} \mathrm{~cm} / \mathrm{s}$ and $\alpha=0.09 \mathrm{~cm}^{-1}$ were obtained. This value of $S$ seems to be a likely value as the effective surface recombination velocity on air exposed $\mathrm{AlGaAs}$ surfaces under medium photoexcitation intensities. The reason why $\alpha$ is the same for different surfaces is because it is primarily determined by the heavy hole effective mass and the valence band offset.

On the other hand, an explanation is needed to the other region of Fig. 6, showing gradual change of PL intensity for values of $d$ from about 30 up to $300 \mathrm{~nm}$. This behavior could not be fitted into the earlier equation, as clearly seen by the dashed curve in Fig. 6. In fact, it cannot be due to tunneling assisted surface recombination, because of the large distance values involved. We believe that this is due to a higher order effect of change of carrier supply from barrier layers as shown previously for InP-based QWRs. ${ }^{13}$ Namely, as the value of the top barrier thickness, $d$, is decreased from a large value, the amount of carriers which are photogenerated within the top barrier layer and subsequently flow into the QWR region is reduced, causing the observed gradual reduction of PL intensity. In other words, the constant $A$ in Eq. (2) is actually a slowing varying function of $d$. The boundary of these two regions should, then, be interpreted as the point where the tunneling assisted surface recombination becomes appreciable on top of the gradual reduction of carrier supply with reduction of $d$. The experimentally observed value of 30 $\mathrm{nm}$ seems to be a reasonable value as a distance for occurrence of appreciable tunneling of holes.

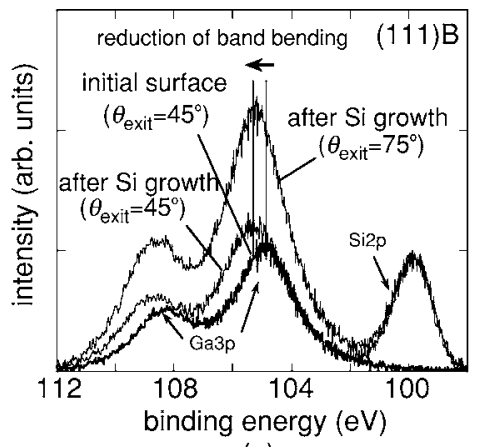

(a)

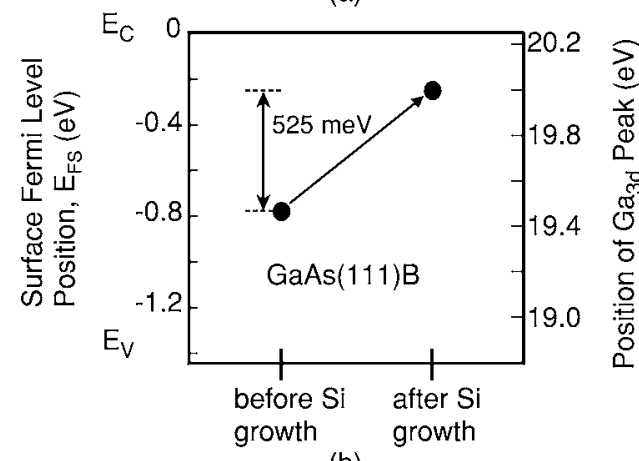

(b)

FIG. 8. (a) XPS spectra from a planar (111)B GaAs surface before and after $\mathrm{Si}$ growth and (b) surface Fermi level position before and after Si growth determined from XPS Ga $3 d$ peak positions.

\section{Effects of Si ICL-based passivation}

Before applying the Si-ICL-based passivation technique on the (111)B surface for the first time in this study, basic XPS measurements were carried out to get information on the growth of the Si layer and on the band bending. Based on the previous finding that the Si-ICL technique is more effective on the Ga-rich surface, ${ }^{14}$ an attempt was also made to deposit a monolayer level Ga before MBE growth. Without $\mathrm{Ga}$ deposition, the surfaces showed well defined $(\sqrt{19}$ $\times \sqrt{19})$ or $(2 \times 2)$ reflection high-energy electron diffraction pattern depending on the temperature in agreement with the previous report. ${ }^{21}$ After Ga supply, the pattern changed to a $(1 \times 1)$ pattern.

XPS spectra taken on a planar (111)B GaAs substrate before and after MBE growth of a Si layer are shown in Fig. 8(a) for various values of the exit angle of photoelectrons, $\theta_{\text {exit }}$. Here, $\theta_{\text {exit }}$ is defined as the angle between the sample surface and the direction of the spectrometer, noting that, out of photoelectrons exiting from the surface, those traveling in the spectrometer direction are detected. As seen in Fig. 8(a), a clear Si $2 p$ peak appeared on the spectra after Si growth, and its shape and height remain unchanged with the change of $\theta_{\text {exit }}$. This indicates that an ultrathin and uniform Si layer is formed on the top of the surface. On the other hand, the height of the neighboring Ga $3 p$ peak changed with $\theta_{\text {exit }}$. This indicates that $\mathrm{Ga}$ atoms are underneath the Si layer, and the peak height reduces with reduction of $\theta_{\text {exit }}$ because the path length of photoelectrons traversing the Si layer is in- 
creased. By analyzing the observed $\theta_{\text {exit }}$ dependence of the Ga $3 p$ peak height, the thickness of the Si layer in this case was estimated to be $1.4 \mathrm{~nm}$.

It was also found that $\mathrm{Si}$ growth caused shifts of binding energy positions of Ga $3 p$, Ga $3 d$, As $3 d$, and Si $2 p$ core level peaks by the same amount of $0.53 \mathrm{eV}$ toward higher binding energies, as seen in Fig. 8(a) for the Ga $3 p$ corelevel. This indicates that the shift is due to a change of the band bending. Using the published data ${ }^{22}$ on the energy difference of the valence band edge and the core level peaks that was determined on the cleaved (110) surface under the flatband condition due to absence of surface states, the absolute positions of the Fermi level in our sample before and after $\mathrm{Si}$ growth were estimated from the XPS peak positions as shown in Fig. 8(b). Before Si deposition, the Fermi level lied at $0.8 \mathrm{eV}$ from the conduction band edge, $E_{C}$, similarly to the (001) surface of $n$-type GaAs. ${ }^{23}$ This indicates that the Fermi level was strongly pinned by a high density of surface states. On the other hand, the position of the Fermi level moved to $E_{C} 0.2 \mathrm{eV}$ after Si growth, corresponding to a large reduction of the band bending. A most likely mechanism for this movement is reduction of surface states. This result indicated that the Si-ICL passivation method is also effective to reduce the density of surface states on the (111)B surface similarly to the case of the (001) surface reported in Ref. 14. As for the effect of $\mathrm{Ga}$ before $\mathrm{Si}$ growth, no marked difference in the final band bending was observed, although it definitely changed the surface stoichiometry and slightly increased the change of band bending. Thus, control of surface stoichiometery may not be so critical on the (111)B surface, as compared with the (001) surface.

Finally, with this promising result, the Si-ICL passivation technique was applied to both the (001) and (111)B QWR samples by growing $\mathrm{Si}$-ICL films on the air exposed surfaces of their top AlGaAs barrier layers after the standard oxide removal procedure in the MBE chamber. In both cases, a monolayer level $\mathrm{Al}$ was deposited prior to Si-ICL deposition.

The PL spectra obtained before and after Si-ICL passivation are compared in Figs. 9(a) and 9(b) for the (001) QWR sample and (111)B QWR sample, respectively. In these samples, the AlGaAs barrier layer was thinned down by anodic etching to such an extent that PL intensities from QWRs were decreased down to about $10 \%$ of the original values at $d=300 \mathrm{~nm}$ and then the Si-ICL-based passivation was applied. Deconvolution of the PL peaks was made in the same way as explained previously. It is seen in Figs. 9(a) and 9(b) that remarkable increase of PL intensity is evident on the deconvoluted QWR peaks in both cases. Namely, after passivation, a four times increase of PL intensity was achieved for the (001) QWR sample, and a three times increase was achieved for the (111)B QWR sample.

The observed improvements are also plotted in Fig. 6 where two white circles and one triangle show the data points obtained on Si-ICL passivated QWR samples. It is seen that these data points lie on the extrapolated portion of the solid curve corresponding to reduction of carrier supply. It strongly suggests that exponential reduction of the PL in-

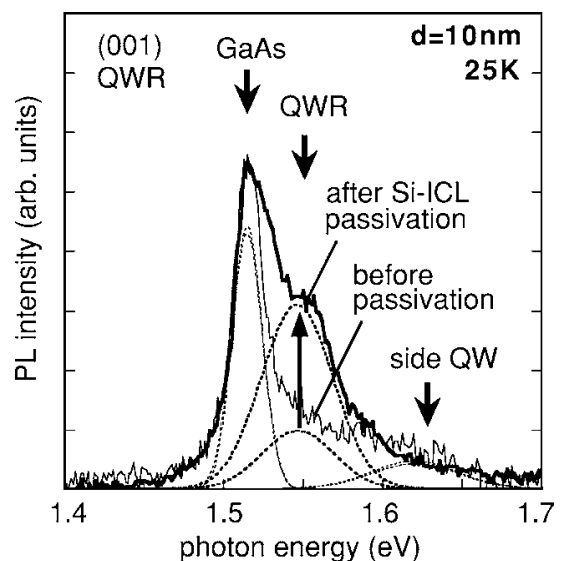

(a)

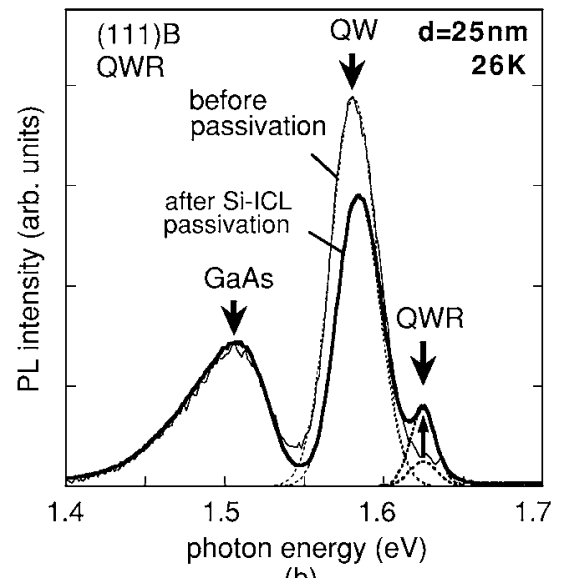

(b)

FIG. 9. PL spectra before and after Si-ICL passivation (a) for a (001) QWR sample and (b) for a (111)B QWR sample.

tensity due to tunneling assisted surface recombination of photogenerated carriers through surface states has been almost completely suppressed by passivation. This suppression can be explained in terms of successful reduction of surface states. Thus, the result obtained in this study seems to give a great promise for future application of this technique for GaAs-based quantum nanoelectronics.

\section{CONCLUSION}

In this article, the effects of surface states and surface passivation on PL properties of GaAs QWRs grown on (001) and (111)B substrates by the selective MBE method were investigated. For surface passivation, the Si-ICL based passivation technique, including MBE growth of ultrathin (about $1 \mathrm{~nm}$ ) Si interlayer was used. In both of the selectively grown QWRs on (001) and (111)B substrates, the PL intensities reduced exponentially with reduction of the wireto-surface distance, $d$, being coexistent with a more gradual reduction due to carrier supply reduction. The rapid exponential reduction was explained in terms of interaction between surface states and quantum confined states, leading to tunneling assisted nonradiative recombination through surface states. Surface passivation by the Si-ICL process almost completely recovered PL intensities not only for QWRs on 
the (001) substrate, but also for QWRs on the (111)B substrate where the technique was applied for the first time.

\section{ACKNOWLEDGMENT}

This work is supported in part by a 21 Century COE program on "Meme-Media Technology Approach to the R\&D of Next-Generation ITs," at Hokkaido University, from MEXT, Japan.

${ }^{1}$ E. Kapon, D. M. Hwang, and R. Bhat, Phys. Rev. Lett. 63, 430 (1989).

${ }^{2}$ T. Fukui, S. Ando, Y. Tokura, and T. Toriyama, Appl. Phys. Lett. 58, 2018 (1991)

${ }^{3}$ H. Hasegawa, H. Fujikura, and H. Okada, MRS Bull. 24, 25 (1999).

${ }^{4}$ T. Sato, I. Tamai, C. Jiang, and H. Hasegawa, IOP Conf. Ser. 170, 325 (2002).

${ }^{5}$ I. Tamai, T. Sato, and H. Hasegawa, Jpn. J. Appl. Phys. 44, 2652 (2005).

${ }^{6}$ H. Hasegawa and S. Kasai, Physica E 11, 149 (2001).

${ }^{7}$ S. Kasai, Y. Amemiya, and H. Hasegawa, Tech. Dig. - Int. Electron Devices Meet. 2000, 585 .

${ }^{8}$ S. Kasai and H. Hasegawa, IEEE Electron Device Lett. 23, 446 (2002).

${ }^{9}$ A. Kameda, S. Kasai, T. Sato, and H. Hasegawa, Solid-State Electron. 47, 323 (2003).
${ }^{10}$ J. M. Moison, K. Elcess, F. Houzay, J. Y. Marzin, J. M. Gerard, F. Barthe, and M. Bensoussan, Phys. Rev. B 41, 12945 (1990).

${ }^{11}$ Z. Sobiesierski, D. I. Westwood, D. A. Woolf, T. Fukui, and H. Hasegawa, J. Vac. Sci. Technol. B 11, 1723 (1993).

${ }^{12}$ A. Forchel, A. Menschig, B. E. Maile, H. Leier, and R. Germann, J. Vac. Sci. Technol. B 9, 444 (1991).

${ }^{13}$ H. Fujikura, S. Kodama, T. Hashizume, and H. Hasegawa, J. Vac. Sci. Technol. B 14, 2888 (1996).

${ }^{14}$ S. Anantathanasarn and H. Hasegawa, Appl. Surf. Sci. 216, 275 (2003).

${ }^{15}$ N. Negoro, S. Anantathanasarn, and H. Hasegawa, J. Vac. Sci. Technol. B 21, 1945 (2003).

${ }^{16}$ T. Sato, I. Tamai, and H. Hasegawa, J. Vac. Sci. Technol. B 22, 2266 (2004).

${ }^{17}$ H. Hasegawa and H. Hartnagel, J. Electrochem. Soc. 123, 713 (1976).

${ }^{18}$ D. E. Aspnes, B. Schwartz, A. A. Studna, L. Derick, and L. A. Koszi, J. Appl. Phys. 48, 3510 (1977).

${ }^{19}$ B. Adamowicz, M. Miczek, K. Ikeya, M. Mutoh, T. Saitoh, H. Fujikura, and H. Hasegawa, Appl. Surf. Sci. 141, 326 (1999).

${ }^{20}$ A. Ahaitouf and A. Bath, Thin Solid Films 342, 136 (1999).

${ }^{21}$ D. K. Biegelsen, R. D. Bringans, J. E. Northrup, and L. E. Swartz, Phys. Rev. Lett. 65, 452 (1990).

${ }^{22}$ R. W. Grant, E. A. Kraut, S. P. Kowalczyk, and J. R. Waldrop, J. Vac. Sci. Technol. B 1, 320 (1983).

${ }^{23}$ Y. Ishikawa, T. Fukui, and H. Hasegawa, J. Vac. Sci. Technol. B 15, 1163 (1997). 